Supporting Information

\title{
Pd nanoparticles anchored on sustainable chitin for phenol hydrogenation to cyclohexanone
}

Chuanchuan Zhao t, Ziyi Zhang t, Yaru Liu f, Ningzhao Shang f, Hai-Jun Wangt, Chun Wang $\ddagger^{*}$, Yongjun Gaoł*

† Key Laboratory of Medicinal Chemistry and Molecular Diagnosis of Ministry of Education, Key Laboratory of Analytical Science and Technology of Hebei Province, College of Chemistry and Environmental Science, Hebei University, Baoding 071002, China.

$\ddagger$ College of Science, Hebei Agricultural University, Baoding 071001, China.

\section{Corresponding Author}

*E-mail: yjgao@hbu.edu.cn ( Yongjun Gao );

*E-mail: wangchun@hebau.ac.cn ( Chun Wang )

Number of pages: 6

Number of figures: 3

Number of tables: 2 
Table S1. ICP-OES measurement results of Pd-chitin-NaBH ${ }_{4}$ and Pd-chitin-150.

\begin{tabular}{ccc}
\hline Entry & Catalyst & $\mathrm{Pd} \%$ \\
\hline 1 & Pd-chitin-NaBH & 1.01 \\
$2^{\mathrm{a}}$ & $\mathrm{Pd}$-chitin-NaBH & 0.79 \\
3 & Pd-chitin-150 & 0.87 \\
$4^{\mathrm{b}}$ & Pd-chitin-150 & 1.04 \\
\hline
\end{tabular}

a. The content of palladium in Pd-chitin- $\mathrm{NaBH}_{4}$ maintains after its fourth use, b. The content of palladium in Pd-chitin-150 maintains after its fifth use.

a)

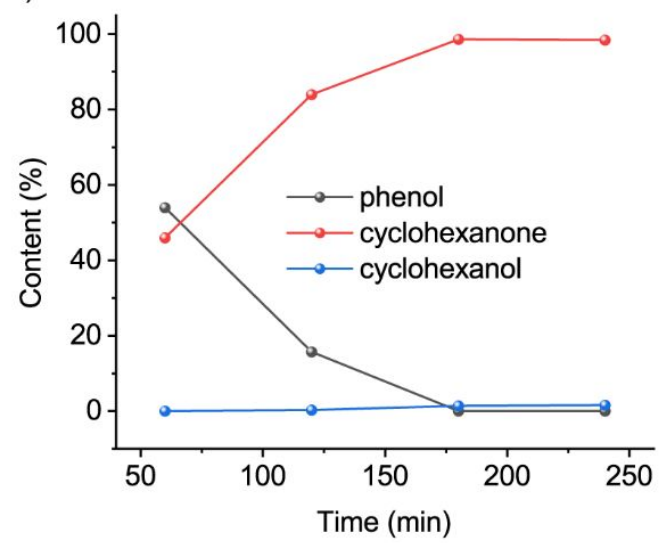

b)

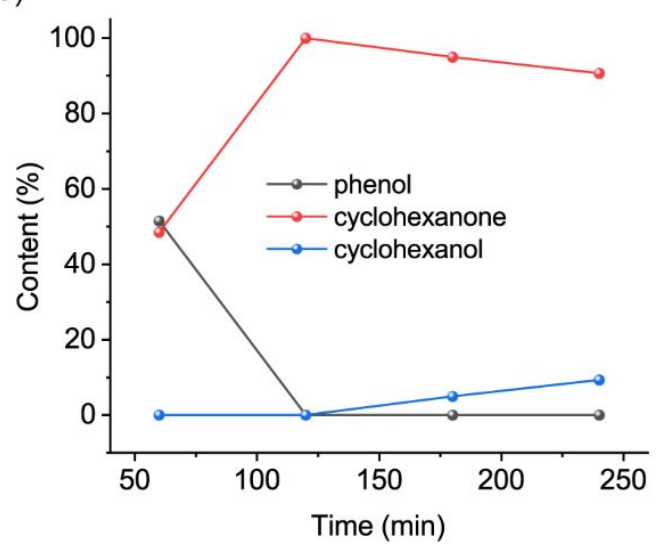


Figure S1. a) Kinetics of phenol hydrogenation with $\mathrm{HCOONa}$ as hydrogen source, b) Kinetics of phenol hydrogenation with $\mathrm{H}_{2}$ as hydrogen source.
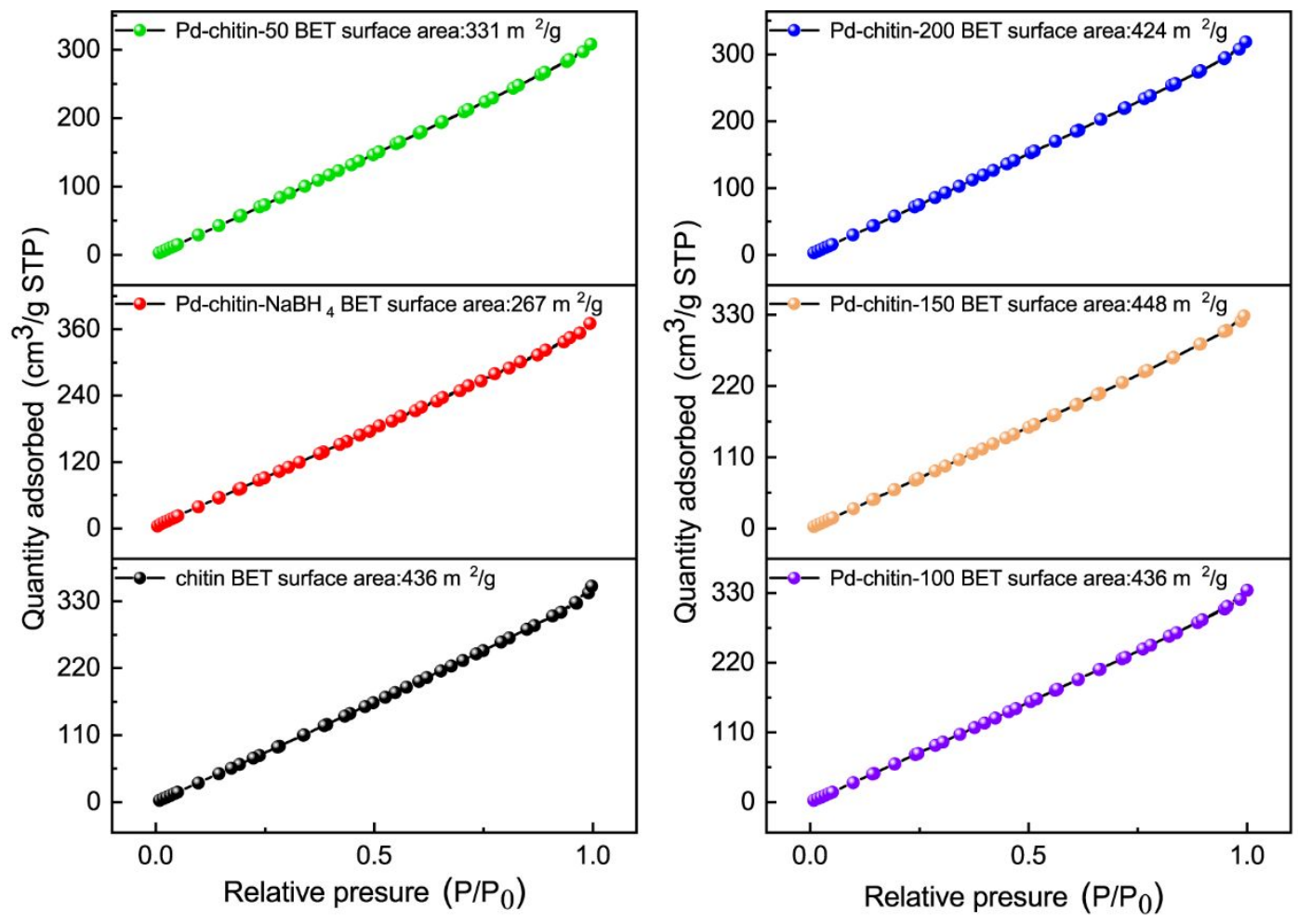

Figure S2. Nitrogen adsorption-desorption isotherms of chitin, $\mathrm{Pd}$-chitin- $\mathrm{NaBH}_{4}, \mathrm{Pd}$-chitin-T. 


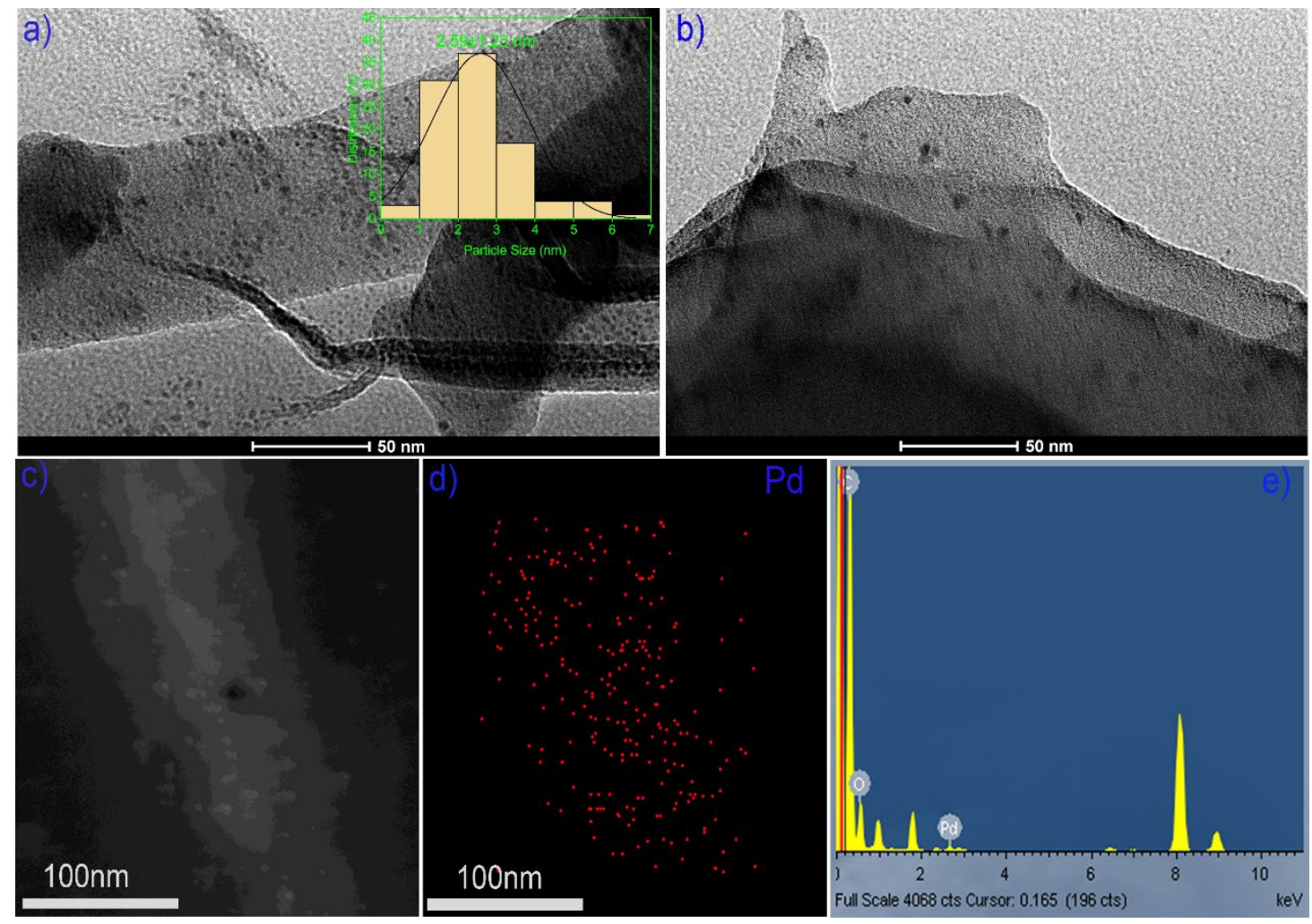

Figure S3. a) b) TEM images of the Pd-chitin- $\mathrm{NaBH}_{4}$ sample, c) d) e) SEM-EDS spectra of Pdchitin-150. 
Table S2. Comparison of catalytic performance of different catalysts for hydrogenate phenol.

\begin{tabular}{|c|c|c|c|c|c|c|c|}
\hline Catalyst & Catalyst amount & $\begin{array}{r}\mathrm{T} \\
\left({ }^{\circ} \mathrm{C}\right)\end{array}$ & $\mathrm{t}[\mathrm{h}]$ & Hydrogen source & $\begin{array}{l}\text { Conv } \\
(\%) .\end{array}$ & $\begin{array}{l}\text { Sel } \\
(\%) .\end{array}$ & Ref \\
\hline Pd-chitin- & $5 \mathrm{~mol} \%$ to & 90 & 3 & HCOONa & 100 & 98.6 & this \\
\hline Pd- & $5.5 \mathrm{~mol} \%$ to & 80 & 5 & HCOONa & 88 & $>99$ & 1 \\
\hline Pd- & $5.5 \mathrm{~mol} \%$ to & 80 & 5 & HCOONa & $>99$ & 54 & 1 \\
\hline Pd- & $5.5 \mathrm{~mol} \%$ to & 80 & 5 & HCOONa & 90 & 97 & 1 \\
\hline $5 \% \mathrm{Rh} @ \mathrm{C}$ & $0.0228 \mathrm{~g}$ & 55 & 2 & $10 \mathrm{MPa} \mathrm{H}_{2}+10$ & 53 & 18 & 2 \\
\hline $5 \% \mathrm{Pd} @ \mathrm{C}$ & $0.1400 \mathrm{~g}$ & 55 & 2 & $10 \mathrm{MPa} \mathrm{H}_{2}+10$ & 3 & 54 & 2 \\
\hline Pd@ML- & $5 \mathrm{~mol} \%$ to & 25 & 11 & $0.1 \mathrm{MPa} \mathrm{H}_{2}$ & 99 & 99 & 2 \\
\hline Pd@mpg- & $4 \mathrm{~mol} \%$ to & 55 & 3 & $0.1 \mathrm{MPa} \mathrm{H}_{2}$ & 44 & $>99$ & 2 \\
\hline $\mathrm{Pd} / \mathrm{C}$ & $0.025 \mathrm{~g}$ & 100 & 2 & $1 \mathrm{MPa} \mathrm{H}_{2}$ & 38 & 82 & 3 \\
\hline $\mathrm{Pd} @ \mathrm{CN}-2$ & $4 \mathrm{~mol} \%$ to & 45 & 4 & $0.1 \mathrm{MPa} \mathrm{H}_{2}$ & 82 & 94 & 4 \\
\hline $\mathrm{Pd} @ \mathrm{MgO}$ & $4 \mathrm{~mol} \%$ to & 55 & 3 & $0.1 \mathrm{MPa} \mathrm{H}_{2}$ & 45 & 98 & 4 \\
\hline $\mathrm{Pd} @ \mathrm{CeO}_{2}$ & $4 \mathrm{~mol} \%$ to & 55 & 3 & $0.1 \mathrm{MPa} \mathrm{H}_{2}$ & 10 & 97 & 4 \\
\hline
\end{tabular}




\section{REFERENCES}

(1) Chen, A.; Li, Y.; Chen, J.; Zhao, G.; Ma, L.; Yu, Y. Selective Hydrogenation of Phenol and

Derivatives over Polymer-Functionalized Carbon-Nanofiber-Supported Palladium Using Sodium Formate as the Hydrogen Source. Chempluschem. 2013, 78, 1370-1378, DOI: 10.1002/cplu.201300238.

(2) Li, Y.; Xu, X.; Zhang, P.; Gong, Y.; Li, H.; Wang, Y. Highly selective Pd@mpg-C ${ }_{3} \mathrm{~N}_{4}$ catalyst for phenol hydrogenation in aqueous phase. RSC Adv. 2013, 3, 10973-10982, DOI: 10.1039/C3RA41397G.

(3) Li, M.; Li, Y.; Jia, L.; Wang, Y. Tuning the selectivity of phenol hydrogenation on Pd/C with acid and basic media. Catal. Commun. 2018, 103, 88-91, DOI: 10.1016/j.catcom.2017.09.028.

(4) Xu, X.; Li, H.; Wang, Y. Selective Hydrogenation of Phenol to Cyclohexanone in Water over Pd@N-Doped Carbon Derived from Ionic-Liquid Precursors. ChemCatChem. 2014, 6, 3328-3332, DOI: 10.1002/cctc.201402561. 